\title{
Synthesis of an L-rhamnose tetrasaccharide, the common and major structure of the repeating unit of the O-antigenic polysaccharide of a strain of Klebsiella pneumoniae and Pseudomonas holci
}

\author{
Jianjun Zhang, Yuliang Zhu, Fanzuo Kong* \\ Research Center for Eco-Environmental Sciences, Academia Sinica, PO Box 2871, Beijing 100085, PR China
}

Received 8 August 2001; accepted 18 September 2001

\begin{abstract}
A tetrasaccharide, $\alpha$-L-Rhap-(1 $\rightarrow 3)-\alpha$-L-Rhap-(1 $\rightarrow 2)-\alpha$-L-Rhap-( $1 \rightarrow 2)$-L-Rhap, the common and major structure of the repeating unit of the O-antigenic polysaccharide of a strain of Klebsiella pneumoniae and Pseudomonas holci was synthesized as its methyl and octyl glycosides. Selective 3-O-glycosylation of allyl $\alpha$-L-rhamnopyranoside with 2,3,4-tri- $O$-acetyl- $\alpha$-L-rhamnopyranosyl trichloroacetimidate gave allyl 2,3,4-tri- $O$-acetyl- $\alpha$-L-rhamnopyranosyl-( $1 \rightarrow$ 3 )- $\alpha$-L-rhamnopyranoside (3). Benzoylation, deallylation, and trichloroacetimidation afforded 2,3,4-tri- $O$-acetyl- $\alpha$-Lrhamnopyranosyl-( $1 \rightarrow 3)$-2,4-di- $O$-benzoyl- $\alpha$-L-rhamnopyranosyl trichloroacetimidate $(6)$. Self condensation of 3,4 -di- $O$-benzoyl- $\beta$-L-rhamnopyranose 1,2 -methyl orthoester or 1,2-octyl orthoester gave methyl or octyl 2 - $O$-acetyl3,4-di- $O$-benzoyl- $\alpha$-L-rhamnopyranosyl-( $1 \rightarrow 2)$-3,4-di- $O$-benzoyl- $\alpha$-L-rhamnopyranoside $(\mathbf{1 6}$ or $\mathbf{1 7})$, and subsequent selective deacetylation gave the disaccharide acceptor (18 or 19). Coupling of 6 with 18 (or 19), followed by deacylation in ammonia-saturated methanol, produced the target tetrasacharide. (C) 2001 Elsevier Science Ltd. All rights reserved.
\end{abstract}

Keywords: Oligosaccharide; Rhamnose; Antigen

The tetrasaccharide, $\alpha$-L-Rhap $-(1 \rightarrow 3)-\alpha$-LRhap $-(1 \rightarrow 2)-\alpha-\mathrm{L}-\mathrm{Rha} p-(1 \rightarrow 2)-\mathrm{L}-\mathrm{Rha} p$, is the common and major structure of the repeating units of the O-antigenic polysaccharide of a strain of Klebsiella pneumoniae strain ${ }^{1}$ and Pseudomonas holci. ${ }^{2}$ The former repeating unit contains one more $\alpha-(1 \rightarrow 2)$-linked L-rhamnose residue at the reducing end, while the

* Corresponding author. Tel.: +86-10-62936613; fax: $+86-10-62923563$.

E-mail address: fzkong@mail.rcees.ac.cn (F. Kong). latter one has a 3-acetylamino-3,6-dideoxy- $\alpha$ D-galactopyranosyl residue attached to the 3$O$-position of the rhamnose unit reducing end. For investigation of the oligosaccharide's structure-bioactivity relationships, we present herewith the synthesis of the target tetrasaccharide.

As outlined in Scheme 1, condensation of 2,3,4-tri- $O$-acetyl- $\alpha$-L-rhamnopyranosyl trichloroacetimidate (2) with unprotected allyl $\alpha$-L-rhamnopyranoside (1) selectively gave allyl 2,3,4-tri- $O$-acetyl- $\alpha$-L-rhamnopyranosyl$(1 \rightarrow 3)$ - $\alpha$-L-rhamnopyranoside (3) in satis- 
factory yield $(64.3 \%)$. Keeping the temperature below $-10^{\circ} \mathrm{C}$ during the addition of TMSOTf was necessary in order to avoid byproduct formation. We rationalized that the good regioselectivity is owing to the formation of 3-O-linked orthoester disaccharide, followed by an intramolecular rearrangement. ${ }^{3}$ The $(1 \rightarrow 3)$ linkage was confirmed by benzoylation of 3 , and the ${ }^{1} \mathrm{H}$ NMR spectrum of the resultant 4 showed a ${ }^{1} \mathrm{H}$ NMR spectrum identical to that reported in the literature. ${ }^{4}$ Compared to our previously reported method for preparation of $\alpha$ - $(1 \rightarrow 3)$-linked rhamnose disaccharide with an unprotected rhamnoside as the acceptor via isolated orthoester intermediate ${ }^{4}$ the present technique is simpler. Deallylation with $\mathrm{PdCl}_{2}$, followed by trichloroacetimidation with $\mathrm{CCl}_{3} \mathrm{CN}$ in the presence of DBU or $\mathrm{K}_{2} \mathrm{CO}_{3},{ }^{5}$ gave the disaccharide donor
6. The disaccharide acceptor was readily prepared by the method of self-condensation of the orthoester 12 or $13 .{ }^{6}$ Thus 3,4-di- $O$-benzoyl- $\beta$-L-rhamnopyranose 1,2-methyl (12) and 1,2 -octyl (13) orthoesters were prepared by the sequential reaction of tri- $O$-acetyl- $\alpha-\mathrm{L}-$ rhamnopyranosyl bromide (7) with methanol and octanol, respectively, then Zemplén deacylation, and conventional benzoylation. Selfcondensation of the orthoesters 12 or $\mathbf{1 3}$ promoted by TMSOTf was carried out readily giving the disaccharides $16(68.8 \%)$ or 17 $(70.0 \%)$, respectively, as the major product and the monosaccharides $14(10.7 \%)$ or $\mathbf{1 5}$ $(11.4 \%)$ as the minor one. This chemoselectivity means that the self-condensation of the orthoesters is indeed an effective method for the preparation of $\alpha-(1 \rightarrow 2)$-linked rhamnose disaccharides. Selective deacetylation ${ }^{7}$ of $\mathbf{1 6}$ or
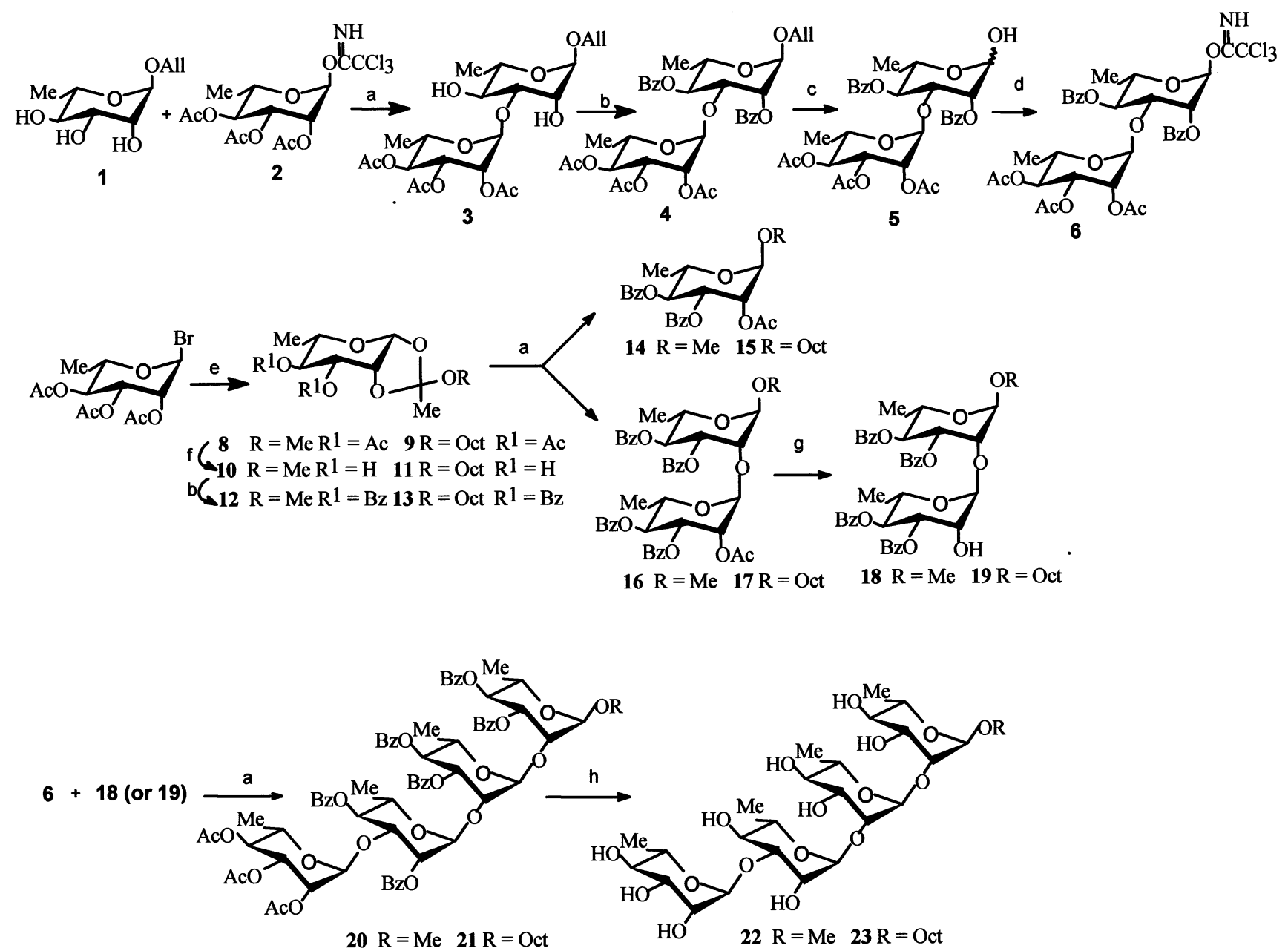

Scheme 1. Reagents and conditions: (a) TMSOTf, $\mathrm{CH}_{2} \mathrm{Cl}_{2}, 4 \AA \mathrm{AS}, \mathrm{N}_{2},-20{ }^{\circ} \mathrm{C}$ to rt, $4 \mathrm{~h}$; (b) $\mathrm{BzCl}-$ pyridine (dry); (c) PdCl , $\mathrm{CH}_{2} \mathrm{Cl}_{2}, 2 \mathrm{~h}$; (d) $\mathrm{CCl}_{3} \mathrm{CN}$, DBU or $\mathrm{K}_{2} \mathrm{CO}_{3}, \mathrm{CH}_{2} \mathrm{Cl}_{2} 8 \mathrm{~h}$; (e) lutidine, $\mathrm{CH}_{2} \mathrm{Cl}_{2}, \mathrm{ROH}, 4 \AA \mathrm{AS}, 4 \mathrm{~h}$; (f) MeONa, MeOH; (g) $\mathrm{CH}_{3} \mathrm{OH}, \mathrm{CH}_{3} \mathrm{COCl}(0.1 \%, \mathrm{v} / \mathrm{v})$; (h) $\mathrm{NH}_{3}, \mathrm{MeOH}$. 
17 with $\mathrm{CH}_{3} \mathrm{COCl}-$ methanol afforded the disaccharide acceptor, methyl 3,4-di- $O$-benoyl- $\alpha$ L-rhamnopyranosyl-( $1 \rightarrow 2)$-3,4-di- $O$-benzoyl$\alpha$-L-rhamnopyranoside (18) or octyl 3,4-di- $O$ benzoyl- $\alpha$-L-rhamnopyranosyl- $(1 \rightarrow 2)$-3,4-di$O$-benzoyl- $\alpha$-L-rhamnopyranoside (19). We were gratified to find that the self-condensation of the orthoester with a long-chain fatty acid alcohol as the aglycone moiety ran smoothly giving the corresponding disaccharide in good yield. This can be an efficient and concise method for the preparation of $\alpha-(1 \rightarrow$ 2)-linked manno- and rhamnopyranose disaccharides with a long-chain fatty acid at the reducing end. With the disaccharide donor 6 and the disaccharide acceptor 18 or 19 at hand, the tetrasaccharide $\mathbf{2 0}$ or $\mathbf{2 1}$ was readily constructed in dichloromethane in the presence of TMSOTf. Finally deacylation of $\mathbf{2 0}$ or 21 in ammonia-saturated methanol gave the target tetrasaccharide $\mathbf{2 2}$ or $\mathbf{2 3}$, and their bioassays are in progress and will be reported in due course.

In summary, a very concise and efficient synthesis of methyl or octyl $\alpha$-L-rhamnopyranosyl-( $(1 \rightarrow 3)-\alpha$-L-rhamnopyranosyl- $(1 \rightarrow 2)-\alpha-\mathrm{L}-$ rhamnopyranosyl- $(1 \rightarrow 2)$ - $\alpha$-L-rhamnopyranoside was achieved in a regio- and stereoselective way. Because of its simplicity and efficiency, this method could be used for construction of higher rhamnose oligosaccharides with $\alpha-(1 \rightarrow 3)$ - and $\alpha-(1 \rightarrow 2)$-linkages.

\section{Experimental}

General methods.-Melting points were determined with a 'Mel-Temp' apparatus. Optical rotations were determined with a Perkin-Elmer model 241-MC automatic polarimeter for solutions in a 1-dm, jacketed cell. ${ }^{1} \mathrm{H}$ NMR spectra were recorded with Varian XL-400 and Varian XL-200 spectrometers, for solutions in $\mathrm{CDCl}_{3}$ with tetramethylsilane $\left(\mathrm{Me}_{4} \mathrm{Si}\right)$ as the internal standard. Chemical shifts are expressed in ppm downfield from the internal $\mathrm{Me}_{4} \mathrm{Si}$ absorption. Mass spectra were recorded with a VG PLATFORM mass spectrometer using the ESI mode. Thin-layer chromatography (TLC) was performed on Silica Gel HF with detection by charring with $30 \%$ (v/v) $\mathrm{H}_{2} \mathrm{SO}_{4}$ in $\mathrm{MeOH}$ or by UV detection. Column chromatography was conducted by elution of a column $(8 \times 100 \mathrm{~mm}, 16 \times 240$ $\mathrm{mm}, 18 \times 300 \mathrm{~mm}, 35 \times 400 \mathrm{~mm}$ ) of silica gel (100-200 mesh) and EtOAc-petroleum ether (bp $60-90^{\circ} \mathrm{C}$ ) as the eluent. Analytical LC was performed with a Gilson HPLC consisting of a pump (model 306), a stainless steel column packed with silica gel (Spherisorb $\mathrm{SiO}_{2}, 10 \times 300 \mathrm{~mm}$ or $\left.4.6 \times 250 \mathrm{~mm}\right)$, a differential refractometer (132-RI Detector), and a UV-vis detector (model 118). EtOAcpetroleum ether (bp $60-90{ }^{\circ} \mathrm{C}$ ) was used as the eluent at a flow rate of $1-4 \mathrm{~mL} / \mathrm{min}$. Solutions were concentrated at a temperature $<60{ }^{\circ} \mathrm{C}$ under diminished pressure.

Allyl 2,3,4-tri-O-acetyl- $\alpha$-L-rhamnopyranosyl-(1 $\rightarrow 3)$-2,4-di-O-benzoyl- $\alpha$-L-rhamnopyranoside (4). - 2,3,4-Tri- $O$-acetyl- $\alpha$-L-rhamnopyranosyl trichloroacetimidate (1) (4.350 g, 10 mmol) and allyl $\alpha$-L-rhamnopyranoside (2) $(2.04 \mathrm{mg}, 10 \mathrm{mmol})$ were dried together under high vacuum for $2 \mathrm{~h}$, then dissolved in anhyd $\mathrm{CH}_{2} \mathrm{Cl}_{2}(40 \mathrm{~mL})$. TMSOTf $(60 \mu \mathrm{L}, 0.2$ equiv) was added dropwise at $-25^{\circ} \mathrm{C}$ with $\mathrm{N}_{2}$ protection. The reaction mixture was stirred for 3 $\mathrm{h}$, during which time the temperature was gradually raised to ambient temperature. Then the mixture was neutralized with triethylamine and concentrated to dryness under reduced pressure to afford the crude allyl 2,3,4-tri$O$-acetyl- $\alpha$ - L-rhamnopyranosyl - $(1 \rightarrow 3)$ - $\alpha$ - Lrhamnopyranoside (3). To the solution of crude 3 in pyridine $(20 \mathrm{~mL}), \mathrm{BzCl}(3.5 \mathrm{~mL}, 30$ $\mathrm{mmol}$ ) was added dropwise, and the mixture was stirred overnight at rt. TLC (3:1 petroleum ether-EtOAc) indicated that the reaction was complete. Ice water was added, and the mixture was diluted with $\mathrm{CH}_{2} \mathrm{Cl}_{2}$, washed with $1 \mathrm{~N} \mathrm{HCl}$, water, and satd aq $\mathrm{NaHCO}_{3}$. The organic layer was combined, dried, and concentrated. Purification of the crude product by column chromatography (3:1 petroleum ether-EtOAc) gave 4 (4.400 g, $64.3 \%$ for two steps $)$ as a syrup: $[\alpha]_{\mathrm{D}}+62^{\circ}(c$ 1.0, $\left.\mathrm{CHCl}_{3}\right)$, lit. ${ }^{4}[\alpha]_{\mathrm{D}}+64^{\circ}\left(c 1.5, \mathrm{CHCl}_{3}\right)$. Compound 4 gave ${ }^{1} \mathrm{H}$ NMR data identical to those reported in the literature. ${ }^{4}$

2,3,4-Tri-O - acetyl - $\alpha$ - L - rhamnopyranosyl$(1 \rightarrow 3)$-2,4-di-O-benzoyl- $\alpha$-L-rhamnopyranosyl 2,2,2-trichloroacetimidate (6).- - To a solution 
of allyl 2,3,4-tri- $O$-acetyl- $\alpha$-L-rhamnopyranosyl-(1 $\rightarrow 3)$-2,4-di- $O$-benzoyl- $\alpha$-L-rhamnopyranoside (4) (684 $\mathrm{mg}, 1 \mathrm{mmol})$ in $90 \% \mathrm{AcOH}$ $(10 \mathrm{~mL})$ containing AcONa $(293 \mathrm{mg}, 3 \mathrm{mmol})$ was added $\mathrm{PdCl}_{2}(89 \mathrm{mg}, 0.5 \mathrm{mmol})$, and the mixture was stirred for $12 \mathrm{~h}$, at the end of which time TLC (2:1 petroleum ether-EtOAc) indicated that the reaction was complete. The mixture was diluted with $\mathrm{CH}_{2} \mathrm{Cl}_{2}(30 \mathrm{~mL})$ and washed with water and satd aq $\mathrm{NaHCO}_{3}$. The organic layer was concentrated, and the residue was passed through a short silica gel column with 2:1 petroleum ether-EtOAc as the eluent to give crude 2,3,4-tri- $O$-acetyl- $\alpha$-Lrhamnopyranosyl - $(1 \rightarrow 3)$ - 2,4 - di - $O$ - benzoyl$\alpha, \beta$-L-rhamnopyranose (5) (620 $\mathrm{mg}, 96 \%)$. Compound 5 was dissolved in $\mathrm{CH}_{2} \mathrm{Cl}_{2}$ (10 $\mathrm{mL})$, and $\mathrm{CCl}_{3} \mathrm{CN}(0.2 \mathrm{ml}, 2 \mathrm{mmol})$ and $\mathrm{DBU}$ $(27 \mu \mathrm{L}, 0.18 \mathrm{mmol})$ were added. The reaction mixture was stirred for $2 \mathrm{~h}$, at the end of which time TLC (2:1 petroleum ether-EtOAc) indicated that the reaction was complete. Concentration of the reaction mixture, followed by purification of the crude product on a silica gel column with 2:1 petroleum ether-EtOAc as the eluent, furnished the disaccharide donor $6(710 \mathrm{mg}, 90 \%)$ as a syrup: $[\alpha]_{\mathrm{D}}+4.3^{\circ}(c 1.3$, $\left.\mathrm{CHCl}_{3}\right) .{ }^{1} \mathrm{H} \mathrm{NMR}\left(\mathrm{CDCl}_{3}\right): \delta 8.80(\mathrm{~s}, 1 \mathrm{H}$, $\left.\mathrm{CNHCCl}_{3}\right), 8.18-7.43(\mathrm{~m}, 10 \mathrm{H}, 2 \mathrm{Bz}-\mathrm{H}), 6.44$ $\left(\mathrm{d}, 1 \mathrm{H}, J_{1,2} 1.9 \mathrm{~Hz}, \mathrm{H}-1\right), 5.62\left(\mathrm{dd}, 1 \mathrm{H}, J_{1,2}\right.$ 1.9, $\left.J_{2,3} 3.2 \mathrm{~Hz}, \mathrm{H}-2\right), 5.59\left(\mathrm{dd}, 1 \mathrm{H}, J_{3,4}=\right.$ $\left.J_{4,5}=9.8 \mathrm{~Hz}, \mathrm{H}-4\right), 5.09\left(\mathrm{dd}, 1 \mathrm{H}, J_{2^{\prime}, 3^{\prime}} 3.2\right.$, $\left.J_{3^{\prime}, 4^{\prime}} 9.8 \mathrm{~Hz}, \mathrm{H}-3^{\prime}\right), 4.94-4.91$ (m, $2 \mathrm{H}, \mathrm{H}^{\prime} 1^{\prime}$, $\left.\mathrm{H}-2^{\prime}\right), 4.89\left(\mathrm{dd}, 1 \mathrm{H}, J_{3^{\prime}, 4^{\prime}}=J_{4^{\prime}, 5^{\prime}}=9.8 \mathrm{~Hz}\right.$, H-4'), 4.49 (dd, $1 \mathrm{H}, J_{2,3} 3.4, J_{3,4} 9.8 \mathrm{~Hz}, \mathrm{H}-3$ ), $4.22(\mathrm{~m}, 1 \mathrm{H}), 3.93(\mathrm{~m}, 1 \mathrm{H}), 1.94(\mathrm{~s}, 3 \mathrm{H}$, $\left.\mathrm{CH}_{3} \mathrm{CO}\right), 1.87$ (s, $\left.3 \mathrm{H}, \mathrm{CH}_{3} \mathrm{CO}\right), 1.82(\mathrm{~s}, 3 \mathrm{H}$, $\mathrm{CH}_{3} \mathrm{CO}$ ), 1.35 (d, $\left.3 \mathrm{H}, J 6.2 \mathrm{~Hz}\right), 1.07$ (d, $3 \mathrm{H}$, $J 6.3 \mathrm{~Hz}$ ). Anal. Calcd for $\mathrm{C}_{34} \mathrm{H}_{36} \mathrm{Cl}_{3} \mathrm{NO}_{14}$ : C, 51.75; H 4.60. Found: C, 51.56; H, 4.58.

3,4-Di-O-acetyl-1,2-O-octoxyethylidene- $\beta$-Lrhamnopyranose (9). - A mixture of 2,3,4-tri$O$-acetyl- $\alpha$-L-rhamnopyranosyl bromide

(2.0 g, $5.7 \mathrm{mmol})$, 2,4-lutidine $(0.8 \mathrm{~mL}, 7.2$ $\mathrm{mmol})$, and tetrabutylammonium bromide $(0.8 \mathrm{~g}, 2.4 \mathrm{mmol})$ in anhyd $\mathrm{CH}_{2} \mathrm{Cl}_{2}(10 \mathrm{~mL})$ was stirred at $\mathrm{rt}$, and $\mathrm{C}_{8} \mathrm{H}_{17} \mathrm{OH}(0.95 \mathrm{~mL}, 6$ mmol) was added. The reaction mixture was stirred for $24 \mathrm{~h}$. TLC (1:1 petroleum etherEtOAc) indicated that the reaction was complete. The reaction mixture was concentrated and subjected to column chromatography to give $9(1.8 \mathrm{~g}, 78.6 \%)$ as a syrup: $[\alpha]_{\mathrm{D}}+13.5^{\circ}$ $\left.(c) 1.2, \mathrm{CHCl}_{3}\right) \cdot{ }^{1} \mathrm{H} \mathrm{NMR}\left(\mathrm{CDCl}_{3}\right): \delta 5.37(\mathrm{~d}, 1$ $\left.\mathrm{H}, J_{1,2} 2.2 \mathrm{~Hz}, \mathrm{H}-1\right), 5.09\left(\mathrm{dd}, 1 \mathrm{H}, J_{2,3} 3.9, J_{3,4}\right.$ $9.9 \mathrm{~Hz}, \mathrm{H}-3), 5.04\left(\mathrm{dd}, 1 \mathrm{H}, J_{3,4}=J_{4,5}=9.9\right.$ $\mathrm{Hz}, \mathrm{H}-4), 4.55$ (dd, $1 \mathrm{H}, J_{1,2} 2.2, J_{2,3} 3.9 \mathrm{~Hz}$, $\mathrm{H}-2), 3.53-3.40$ (m, $3 \mathrm{H}, \mathrm{H}-5, \mathrm{OCH}_{2} \mathrm{C}_{7} \mathrm{H}_{15}$ ), 2.09 (s, $\left.3 \mathrm{H}, \mathrm{CH}_{3} \mathrm{CO}\right), 2.04$ (s, $3 \mathrm{H}, \mathrm{CH}_{3} \mathrm{CO}$ ), $1.71\left(\mathrm{~s}, 3 \mathrm{H}, \mathrm{CH}_{3} \mathrm{CO}_{3}\right), 1.53-0.84(\mathrm{~m}, 18 \mathrm{H}$, $\left.\mathrm{H}-6, \mathrm{C}_{7} \mathrm{H}_{15} \mathrm{CH}_{2} \mathrm{O}\right) ;{ }^{13} \mathrm{C}$ NMR $\left(\mathrm{CDCl}_{3}, 100\right.$ $\mathrm{MHz}): \delta$ 14.1, 17.6, 20.8, 20.8, 22.7, 24.8, 26.1, $29.2,29.3,29.5,31.6,62.7,69.2,70.5,70.8$, 76.6, 97.2, 124.2, 169.8, 169.8, 170.5. Anal. Calcd for $\mathrm{C}_{20} \mathrm{H}_{34} \mathrm{O}_{8}$ : C, 59.68; H, 8.52. found: C, 59.56; H, 8.50.

3,4-Di-O-benzoyl-1,2-O-methoxyethylidene$\beta$-L-rhamnopyranose (12)._- A mixture of 2,3,4-tri- $O$-acetyl- $\alpha$-L-rhamnopyranosyl bromide (7) (2.0 g, $5.7 \mathrm{mmol})$, 2,4-lutidine (0.8 $\mathrm{mL}, \quad 7.2 \mathrm{mmol})$, and tetrabutylammonium bromide $(0.8 \mathrm{~g}, 2.4 \mathrm{mmol})$ in $\mathrm{CH}_{2} \mathrm{Cl}_{2}(1 \mathrm{~mL})$ was stirred at $\mathrm{rt}$, and anhyd $\mathrm{MeOH}(0.5 \mathrm{~mL}$, $12 \mathrm{mmol})$ was added. The reaction mixture was stirred for $24 \mathrm{~h}$. TLC (1:1 petroleum ether-EtOAc) indicated that the reaction was complete. The reaction mixture was concentrated and subjected to column chromatography to give crude product 8 (1.6 g, 93\%). According to the standard method, compound 8 (1.5 g, $4.9 \mathrm{mmol})$ was deacetylated with $\mathrm{MeONa}$ in $\mathrm{MeOH}$ to give 10, and then $\mathbf{1 0}$ was benzoylated with $\mathrm{BzCl}$ in pyridine to furnish 12 in quantitative yield. Flash chromatography (3:1 petroleum ether-EtOAc) of the residue gave pure $12(1.98 \mathrm{~g}, 93.8 \%)$ as a syrup: $[\alpha]_{\mathrm{D}}+100.9^{\circ}\left(c 1.3, \mathrm{CHCl}_{3}\right) \cdot{ }^{1} \mathrm{H} \mathrm{NMR}$ $\left(\mathrm{CDCl}_{3}\right): \delta 8.01-7.36(\mathrm{~m}, 10 \mathrm{H}, 2 \mathrm{Bz}-\mathrm{H})$, 5.54-5.45 (m, $3 \mathrm{H}), 4.82\left(\mathrm{dd}, 1 \mathrm{H}, J_{1,2} 2.5\right.$, $\left.J_{2,3} 3.6 \mathrm{~Hz}, \mathrm{H}-2\right), 3.66$ (m, $\left.1 \mathrm{H}, \mathrm{H}-5\right), 3.26$ (s, 3 $\left.\mathrm{H}, \mathrm{OCH}_{3}\right), 1.79$ (s, $\left.3 \mathrm{H}, \mathrm{CCH}_{3}\right), 0.89$ (d, J $J_{5,6}$ $6.6 \mathrm{~Hz}, 3 \mathrm{H}, \mathrm{H}-6)$. Anal. Calcd for $\mathrm{C}_{23} \mathrm{H}_{24} \mathrm{O}_{8}$ : C, 64.48; H, 5.65. Found: C, 64.29; H, 5.66. 3,4-Di-O-benzoyl-1,2-O-octoxyethylidene- $\beta$ L-rhamnopyranose (13). - According to the standard method, compound $9(2.0 \mathrm{~g}, 5.0$ mmol) was deacetylated with $\mathrm{MeONa}$ in $\mathrm{MeOH}$ to give 11, and then 11 was benzoylated with $\mathrm{BzCl}$ in pyridine to furnish 13 in quantitative yield. Flash chromatography (4:1 petroleum ether-EtOAc) of the residue gave pure 13 as a syrup $(2.5 \mathrm{~g}, 95 \%):[\alpha]_{\mathrm{D}}+91.2^{\circ}$ 
(c 1.3, $\left.\mathrm{CHCl}_{3}\right) .{ }^{1} \mathrm{H}$ NMR $\left(\mathrm{CDCl}_{3}\right): \delta 8.01-$ $7.36(\mathrm{~m}, 10 \mathrm{H}, 2 \mathrm{Bz}-\mathrm{H}), 5.54-5.46(\mathrm{~m}, 3 \mathrm{H}$, $\mathrm{H}-1, \mathrm{H}-3, \mathrm{H}-4), 4.80$ (dd, $1 \mathrm{H}, J_{1,2} 2.5, J_{2,3} 3.6$ $\mathrm{Hz}, \mathrm{H}-2), 3.76(\mathrm{~m}, 1 \mathrm{H}, \mathrm{H}-5), 3.46(\mathrm{~m}, 2 \mathrm{H}$, $\left.\mathrm{OCH}_{2} \mathrm{C}_{7} \mathrm{H}_{15}\right), 1.79\left(\mathrm{~s}, 3 \mathrm{H}, \mathrm{CCH}_{3}\right), 1.52-0.83$ $\left(\mathrm{m}, 18 \mathrm{H}, \mathrm{H}-6, \mathrm{OCH}_{2} \mathrm{C}_{7} H_{15}\right)$. Anal. Calcd for $\mathrm{C}_{30} \mathrm{H}_{38} \mathrm{O}_{8}: \mathrm{C}, 68.42 ; \mathrm{H}, 7.27$. Found $\mathrm{C}, 68.45$; $\mathrm{H}, 7.32$.

Methyl 2-O-acetyl-3,4-di-O-benzoyl- $\alpha$-Lrhamnopyranosyl- $(1 \rightarrow 2)-3,4-d i$-O-benzoyl- $\alpha$ L-rhamnopyranoside (16). - To a solution of compound 12 (856 $\mathrm{mg}, 2 \mathrm{mmol}$ ) in anhyd $\mathrm{CH}_{2} \mathrm{Cl}_{2}(20 \mathrm{~mL})$ was added TMSOTf $(18 \mu \mathrm{L}$, $0.1 \mathrm{mmol}$ ) in the presence of $4 \AA$ molecular sieves under an $\mathrm{N}_{2}$ atmosphere at $-20^{\circ} \mathrm{C}$. The mixture was slowly raised to $\mathrm{rt}$ and stirred for $2 \mathrm{~h}$, neutralized with $\mathrm{Et}_{3} \mathrm{~N}$ and filtered, then the filtrate was concentrated. Purification of the product by column chromatography (3:1 petroleum ether-EtOAc) gave syrupy $\mathbf{1 6}$ $(538 \mathrm{mg}, 68.8 \%)$ as the main product and a syrupy 14 (46 $\mathrm{mg}, 10.7 \%)$ as the minor product. For compound 16: $[\alpha]_{\mathrm{D}}+53^{\circ}(c 1.3$, $\left.\mathrm{CHCl}_{3}\right) .{ }^{1} \mathrm{H}$ NMR $\left(\mathrm{CDCl}_{3}\right): \delta 8.00-7.35(\mathrm{~m}$, $20 \mathrm{H}, 4 \mathrm{Bz}-\mathrm{H}), 5.80(\mathrm{dd}, 1 \mathrm{H}, J 3.3, J 9.8 \mathrm{~Hz})$, $5.76(\mathrm{dd}, 1 \mathrm{H}, J 3.1, J 10.0 \mathrm{~Hz}), 5.66(\mathrm{dd}, 1 \mathrm{H}$, $\left.J_{1^{\prime}, 2^{\prime}} 1.8, J_{2^{\prime}, 3^{\prime}} 3.1 \mathrm{~Hz}, \mathrm{H}-2^{\prime}\right), 5.63(\mathrm{dd}, 1 \mathrm{H}, J$ $9.8 \mathrm{~Hz}), 5.55(\mathrm{dd}, 1 \mathrm{H}, J 9.8 \mathrm{~Hz}), 4.97(\mathrm{~d}, 1 \mathrm{H}$, $J 1.7 \mathrm{~Hz}), 4.86(\mathrm{~d}, 1 \mathrm{H}, J 1.8 \mathrm{~Hz}), 4.30-4.24$ $(\mathrm{m}, 2 \mathrm{H}), 4.10(\mathrm{~m}, 1 \mathrm{H}), 3.49\left(\mathrm{~s}, 3 \mathrm{H}, \mathrm{OCH}_{3}\right)$, $2.04\left(\mathrm{~s}, 3 \mathrm{H}, \mathrm{COCH}_{3}\right), 1.40(\mathrm{~d}, 3 \mathrm{H}, J 6.3 \mathrm{~Hz})$, $1.32(\mathrm{~d}, 3 \mathrm{H}, J 6.4 \mathrm{~Hz})$. Anal. Calcd for $\mathrm{C}_{43} \mathrm{H}_{42} \mathrm{O}_{14}$ : C, 65.97; H, 5.41. Found: C, 65.76; $\mathrm{H}$, 5.38. For compound 14: $[\alpha]_{\mathrm{D}}+55.1^{\circ}(\mathrm{c}$ 1.3, $\left.\mathrm{CHCl}_{3}\right) .{ }^{1} \mathrm{H}$ NMR $\left(\mathrm{CDCl}_{3}\right): \delta 7.98-7.34$ $(\mathrm{m}, 10 \mathrm{H}, 2 \mathrm{Bz}-\mathrm{H}), 5.69\left(\mathrm{dd}, 1 \mathrm{H}, J_{2,3} 3.5, J_{3,4}\right.$ $10.1 \mathrm{~Hz}, \mathrm{H}-3), 5.54\left(\mathrm{dd}, 1 \mathrm{H}, J_{3,4}=J_{4,5}=10.1\right.$ $\mathrm{Hz}, \mathrm{H}-4), 5.46\left(\mathrm{dd}, 1 \mathrm{H}, J_{1,2} 1.6, J_{2,3} 3.5 \mathrm{~Hz}\right.$, $\mathrm{H}-2), 4.75\left(\mathrm{~d}, 1 \mathrm{H}, J_{1,2} 1.6 \mathrm{~Hz}, \mathrm{H}-1\right), 4.11(\mathrm{~m}$, $1 \mathrm{H}, \mathrm{H}-5), 3.47\left(\mathrm{~s}, 3 \mathrm{H}, \mathrm{OCH}_{3}\right), 2.16(\mathrm{~s}, 3 \mathrm{H}$, $\left.\mathrm{CH}_{3} \mathrm{CO}\right), 1.34$ (d, $\left.3 \mathrm{H}, J_{5,6} 6.3 \mathrm{~Hz}, \mathrm{H}-6\right)$. Anal. Calcd for $\mathrm{C}_{23} \mathrm{H}_{24} \mathrm{O}_{8}: \mathrm{C}, 64.48 ; \mathrm{H}, 5.65$. Found: C, 64.32; H, 5.64.

Octyl 2-O-acetyl-3,4-di-O-benzoyl- $\alpha-\mathrm{L}-$ rhamnopyranosyl- $(1 \rightarrow 2)-3,4-d i$-O-benzoyl- $\alpha$ L-rhamnopyranoside (17). - To a solution of compound 13 (1.052 g, $2 \mathrm{mmol})$ in anhyd $\mathrm{CH}_{2} \mathrm{Cl}_{2}(20 \mathrm{~mL})$ was added TMSOTf $(18 \mu \mathrm{L}$, $0.1 \mathrm{mmol}$ ) in the presence of $4 \AA$ molecular sieves under an $\mathrm{N}_{2}$ atmosphere at $-20{ }^{\circ} \mathrm{C}$. The mixture was slowly raised to $\mathrm{rt}$ and stirred for $2 \mathrm{~h}$, neutralized with $\mathrm{Et}_{3} \mathrm{~N}$ and filtered, then the filtrate was concentrated. Purification of the product by column chromatography (5:1 petroleum ether-EtOAc) gave a syrup $\mathbf{1 7}$ $(616 \mathrm{mg}, 70.0 \%)$ as the main product and a syrupy $15(60 \mathrm{mg}, 11.4 \%)$ as the minor product. For compound 17: $[\alpha]_{\mathrm{D}}+62^{\circ}(c 1.0$, $\left.\mathrm{CHCl}_{3}\right) .{ }^{1} \mathrm{H}$ NMR $\left(\mathrm{CDCl}_{3}\right): \delta 8.03-7.33(\mathrm{~m}$, $20 \mathrm{H}, 4 \mathrm{Bz}-\mathrm{H}), 5.85(\mathrm{dd}, 1 \mathrm{H}, J 3.4, J 10.0$ $\mathrm{Hz}), 5.77(\mathrm{dd}, 1 \mathrm{H}, J 3.2, J 10.0 \mathrm{~Hz}), 5.68(\mathrm{dd}$, $\left.1 \mathrm{H}, J_{1^{\prime}, 2^{\prime}} 1.8, J_{2^{\prime}, 3^{\prime}} 3.1 \mathrm{~Hz}, \mathrm{H}-2^{\prime}\right), 5.63(\mathrm{dd}, 1 \mathrm{H}$, $J 9.8 \mathrm{~Hz}), 5.54(\mathrm{dd}, 1 \mathrm{H}, J 9.8 \mathrm{~Hz}), 4.98(\mathrm{~d}, 1$ $\mathrm{H}, J 1.7 \mathrm{~Hz}), 4.95(\mathrm{~d}, 1 \mathrm{H}, J 1.8 \mathrm{~Hz}), 4.28(\mathrm{~m}$, $2 \mathrm{H}), 4.11(\mathrm{~m}, 1 \mathrm{H}), 3.65(\mathrm{~m}, 2 \mathrm{H}), 2.05(\mathrm{~s}, 3$ $\left.\mathrm{H}, \mathrm{COCH}_{3}\right), 1.70-1.32(\mathrm{~m}, 21 \mathrm{H}) ;{ }^{13} \mathrm{C} \mathrm{NMR}$ $\left(\mathrm{CDCl}_{3}, 100 \mathrm{MHz}\right): \delta 14.1,17.6,17.8,20.8$, $22.7,26.2,29.3,29.5,29.5,31.9,66.9,67.5$, $68.3,69.7,69.9,71.3,71.7,72.1,98.7,99.7$, 165.2, 165.4, 165.7, 165.8, 169.8. Anal. Calcd for $\mathrm{C}_{50} \mathrm{H}_{56} \mathrm{O}_{14}: \mathrm{C}, 68.17 ; \mathrm{H}, 6.41$. Found: $\mathrm{C}$, 68.33; H, 6.39. For compound 15: $[\alpha]_{\mathrm{D}}+15^{\circ}$ (c $\left.0.3, \mathrm{CHCl}_{3}\right) .{ }^{1} \mathrm{H}$ NMR $\left(\mathrm{CDCl}_{3}\right): \delta 7.98-$ $7.34(\mathrm{~m}, 10 \mathrm{H}, 2 \mathrm{Bz}-\mathrm{H}), 5.71\left(\mathrm{dd}, 1 \mathrm{H}, J_{2,3} 3.5\right.$, $\left.J_{3,4} 10.1 \mathrm{~Hz}, \mathrm{H}-3\right), 5.54\left(\mathrm{dd}, 1 \mathrm{H}, J_{3,4}=J_{4,5}=\right.$ $10.1 \mathrm{~Hz}, \mathrm{H}-4), 5.46\left(\mathrm{dd}, 1 \mathrm{H}, J_{1,2} 1.7, J_{2,3} 3.4\right.$ $\mathrm{Hz}, \mathrm{H}-2), 4.85\left(\mathrm{~d}, 1 \mathrm{H}, J_{1,2} 1.7 \mathrm{~Hz}, \mathrm{H}-1\right), 4.12$ (m,1 H, H-5), 3.64-3.60 (m, $2 \mathrm{H}), 2.15$ (s, 3 $\left.\mathrm{H}, \mathrm{CH}_{3} \mathrm{CO}\right), 1.70-0.88(\mathrm{~m}, 18 \mathrm{H}) \cdot{ }^{13} \mathrm{C} \mathrm{NMR}$ $\left(\mathrm{CDCl}_{3}, 100 \mathrm{MHz}\right): \delta 14.2,17.7,21.0,22.7$, $26.2,29.4,29.4,29.5,29.5,31.9,66.6,68.5$, $70.1,70.4,71.8,97.6,165.5,165.8,170.1$. Anal. Calcd for $\mathrm{C}_{30} \mathrm{H}_{38} \mathrm{O}_{8}: \mathrm{C}, 68.42 ; \mathrm{H}, 7.27$. Found: C, 68.17; H, 7.25.

Methyl 3,4-di-O-benzoyl- $\alpha$-L-rhamnopyranosyl-(1 $\rightarrow 2)$-3,4-di-O-benzoyl- $\alpha$-L-rhamnopyranoside (18). - To a solution of 16 (782 $\mathrm{mg}, 1$ $\mathrm{mmol})$ in anhyd $\mathrm{MeOH}(50 \mathrm{~mL})$ was added acetyl chloride $(1.5 \mathrm{~mL})$ at $0{ }^{\circ} \mathrm{C}$. The solution was sealed in a flask and stirred at rt until TLC (2:1 petroleum ether-EtOAc) showed that the starting material disappeared. The solution was neutralized with $\mathrm{Et}_{3} \mathrm{~N}$, then concentrated to dryness. The residue was passed through a short silica gel column to give $\mathbf{1 8}$ (688 mg, 93.0\%): $[\alpha]_{\mathrm{D}}+99^{\circ}\left(c 1.6, \mathrm{CHCl}_{3}\right)$. ${ }^{1} \mathrm{H}$ NMR $\left(\mathrm{CDCl}_{3}\right): \delta 7.98-7.36(\mathrm{~m}, 20 \mathrm{H}, 4$ Bz-H), 5.73-5.69 (m, 2 H), 5.64-5.58 (m, 2 H), $5.05(\mathrm{~d}, 1 \mathrm{H}, J 1.4 \mathrm{~Hz}), 4.89(\mathrm{~d}, 1 \mathrm{H}, J 1.5$ $\mathrm{Hz}), 4.45(\mathrm{dd}, 1 \mathrm{H}, J 1.5, J 3.4 \mathrm{~Hz}), 4.33(\mathrm{dd}$, $1 \mathrm{H}, J 1.4, J 3.3 \mathrm{~Hz}), 4.30-4.07(\mathrm{~m}, 2 \mathrm{H}), 3.48$ (s, $3 \mathrm{H}, \mathrm{CH}_{3} \mathrm{O}$ ), 2.20-1.80 (br, $1 \mathrm{H}, \mathrm{OH}$ ), 
1.40-1.30 (m, $6 \mathrm{H})$. Anal. Calcd for $\mathrm{C}_{41} \mathrm{H}_{40} \mathrm{O}_{13}: \mathrm{C}, 66.48 ; \mathrm{H}$, 5.44. Found: C, 66.59; $\mathrm{H}, 5.46$.

Octyl 3,4-di-O-benzoyl- $\alpha$-L-rhamnopyranosyl-(1 $\rightarrow 2)-3,4$-di-O-benzoyl- $\alpha$-L-rhamnopyranoside (19). - To a solution of $17(880 \mathrm{mg}, 1$ $\mathrm{mmol})$ in anhyd $\mathrm{MeOH}(50 \mathrm{~mL})$ was added acetyl chloride $(1.5 \mathrm{~mL})$ at $0{ }^{\circ} \mathrm{C}$. The solution was sealed in a flask and stirred at rt until TLC (3:1 petroleum ether-EtOAc) showed that the starting material disappeared. The solution was neutralized with $\mathrm{Et}_{3} \mathrm{~N}$, then concentrated to dryness. The residue was passed through a short silica gel column to give 19 (730 mg, 87.1\%): $[\alpha]_{\mathrm{D}}+79^{\circ}\left(\mathrm{c} 1.3, \mathrm{CHCl}_{3}\right)$. ${ }^{1} \mathrm{H}$ NMR $\left(\mathrm{CDCl}_{3}\right): \delta 8.01-7.36(\mathrm{~m}, 20 \mathrm{H}, 4$ $\mathrm{Bz}-\mathrm{H}), 5.76-5.70(\mathrm{~m}, 2 \mathrm{H}), 5.62-5.55(\mathrm{~m}, 2$ H), $5.04(\mathrm{~d}, 1 \mathrm{H}, J 1.5 \mathrm{~Hz}), 4.96(\mathrm{~d}, 1 \mathrm{H}, J 1.5$ $\mathrm{Hz}), 4.43$ (dd, $1 \mathrm{H}, J 1.5, J 3.5 \mathrm{~Hz}), 4.32$ (dd, $1 \mathrm{H}, J 1.5, J 3.3 \mathrm{~Hz}), 4.12(\mathrm{~m}, 1 \mathrm{H}), 3.65(\mathrm{~m}$, $2 \mathrm{H}), 1.68-0.88(\mathrm{~m}, 21 \mathrm{H}) ;{ }^{13} \mathrm{C} \mathrm{NMR}\left(\mathrm{CDCl}_{3}\right.$, $100 \mathrm{MHz}): \delta 14.1,17.6,17.7,22.7,26.2,29.3$, $29.4,29.5,31.9,66.7,67.4,68.2,69.7,71.5$, $71.7,72.0,72.2,98.8,101.4,165.3,165.6$, 165.8, 165.9. Anal. Calcd for $\mathrm{C}_{48} \mathrm{H}_{54} \mathrm{O}_{13}$ : C, 68.72; H, 6.49. Found: C, 68.85; H, 6.48.

Methyl 2,3,4-tri-O-acetyl- $\alpha$-L-rhamnopyranosyl-( $1 \rightarrow 3)$-2,4-di-O-benzoyl- $\alpha$-L-rhamnopyranosyl- $(1 \rightarrow 2)-3,4$-di-O-benzoyl- $\alpha$-L-rhamnopyranosyl - $(1 \rightarrow 2)$ - 3,4 - di - O - benzoyl - $\alpha$ - Lrhamnopyranoside (20). - The disaccharide donor 6 (788 $\mathrm{mg}, 1 \mathrm{mmol})$ and the disaccharide acceptor 18 (740 mg, $1 \mathrm{mmol})$ were dried together under high vacuum for $2 \mathrm{~h}$, then dissolved in anhyd $\mathrm{CH}_{2} \mathrm{Cl}_{2}(10 \mathrm{~mL})$. TMSOTf (15 $\mu \mathrm{L}, 0.08$ equiv) was added dropwise at $-20{ }^{\circ} \mathrm{C}$ with $\mathrm{N}_{2}$ protection. The reaction mixture was stirred for $2 \mathrm{~h}$, during which time the reaction temperature was gradually allowed to rise to ambient temperature. Then the mixture was neutralized with triethylamine and concentrated to an oily residue. Purification by column chromatography $(2: 1$ petroleum ether-EtOAc) gave $20(970 \mathrm{mg}, 71.0 \%):[\alpha]_{\mathrm{D}}$ $+94^{\circ}\left(c\right.$ 1.9, $\left.\mathrm{CHCl}_{3}\right) .{ }^{1} \mathrm{H}$ NMR $\left(\mathrm{CDCl}_{3}\right): \delta$ 8.06-7.33 (m, $30 \mathrm{H}, 6 \mathrm{Bz}-\mathrm{H}), 5.92(\mathrm{dd}, 1 \mathrm{H}, J$ $3.2, J 10.0 \mathrm{~Hz}), 5.67-5.48(\mathrm{~m}, 4 \mathrm{H}), 5.40(\mathrm{dd}$, $1 \mathrm{H}, J 9.9 \mathrm{~Hz}), 5.18(\mathrm{~d}, 1 \mathrm{H}, J 1.6 \mathrm{~Hz}, \mathrm{H}-1)$, $5.11(\mathrm{dd}, 1 \mathrm{H}, J 3.1, J 10.1 \mathrm{~Hz}), 5.02-4.92(\mathrm{~m}$, $5 \mathrm{H}), 4.48-4.41(\mathrm{~m}, 3 \mathrm{H}), 4.30(\mathrm{~m}, 1 \mathrm{H}), 4.18$ $(\mathrm{m}, 1 \mathrm{H}), 4.01(\mathrm{~m}, 1 \mathrm{H}), 3.94(\mathrm{~m}, 1 \mathrm{H}), 3.57$ (s, $\left.3 \mathrm{H}, \mathrm{OCH}_{3}\right), 1.98$ (s, $\left.3 \mathrm{H}, \mathrm{CH}_{3} \mathrm{CO}\right), 1.95$ (s, 3 $\mathrm{H}, \mathrm{CH}_{3} \mathrm{CO}$ ), 1.89 (s, $\left.3 \mathrm{H}, \mathrm{CH}_{3} \mathrm{CO}\right), 1.49$ (d, 3 $\mathrm{H}, J 6.1 \mathrm{~Hz}), 1.44(\mathrm{~d}, 3 \mathrm{H}, J 6.1 \mathrm{~Hz}), 1.11$ (d, $3 \mathrm{H}, J 6.1 \mathrm{~Hz}), 1.01(\mathrm{~d}, 3 \mathrm{H}, J 6.1 \mathrm{~Hz}) ;{ }^{13} \mathrm{C}$ NMR $\left(\mathrm{CDCl}_{3}, 100 \mathrm{MHz}\right): \delta 17.1,17.3,17.6$, $17.7,20.5,20.5,20.7,55.2,66.6,67.2,67.5$, $67.6,68.5,69.5,70.5,71.1,71.1,71.6,71.8$, $71.9,71.9,73.1,74.6,76.7,77.7,99.1,99.3$, $100.0,100.8,165.3,165.4,165.4,165.5,165.7$, 165.9, 169.2, 169.3, 170.0. Anal. Calcd for $\mathrm{C}_{73} \mathrm{H}_{74} \mathrm{O}_{26}$ : C, 64.12; H, 5.46. Found: C, 64.32; $\mathrm{H}, 5.45$.

Octyl 2,3,4-tri-O-acetyl- $\alpha$-L-rhamnopyranosyl-(1 $\rightarrow 3)$-2,4-di-O-benzoyl- $\alpha$ - L-rhamnopyranosyl- $(1 \rightarrow 2)$-3,4-di-O-benzoyl- $\alpha$-L-rhamnopyranosyl-( $\rightarrow 2)-3$,4-di-O-benzoyl- $\alpha$-L-rhamnopyranoside (21). - The disaccharide donor 6 (788 $\mathrm{mg}, 1 \mathrm{mmol})$ and the disaccharide acceptor $19(838 \mathrm{mg}, 1 \mathrm{mmol})$ were dried together under high vacuum for $2 \mathrm{~h}$, then dissolved in anhyd $\mathrm{CH}_{2} \mathrm{Cl}_{2}(10 \mathrm{~mL})$. TMSOTf $(15 \mu \mathrm{L}, 0.08$ equiv) was added dropwise at $-20^{\circ} \mathrm{C}$ with $\mathrm{N}_{2}$ protection. The reaction mixture was stirred for $2 \mathrm{~h}$, during which time the reaction temperature was gradually allowed to rise to ambient temperature. Then the mixture was neutralized with triethylamine and concentrated to an oily residue. Purification by column chromatography $(2: 1$ petroleum ether-EtOAc) gave $21(1.140 \mathrm{~g}, 77.9 \%):[\alpha]_{\mathrm{D}}$ $+74^{\circ}\left(c 1.0, \mathrm{CHCl}_{3}\right) \cdot{ }^{1} \mathrm{H} \mathrm{NMR}\left(\mathrm{CDCl}_{3}\right): \delta$ 8.06-7.33 (m, 30 H, 6 Bz-H), 5.87 (dd, $1 \mathrm{H}, J$ $3.2, J 10.2 \mathrm{~Hz}), 5.65-5.58(\mathrm{~m}, 3 \mathrm{H}), 5.45(\mathrm{dd}$, $1 \mathrm{H}, J$ 1.4, J $3.3 \mathrm{~Hz}), 5.29(\mathrm{dd}, 1 \mathrm{H}, J 10.2$ $\mathrm{Hz}), 5.10$ (d, $1 \mathrm{H}, J 1.3 \mathrm{~Hz}), 5.04$ (dd, $1 \mathrm{H}, J$ 3.4, J 9.8 Hz), 4.94-4.88 (m, $4 \mathrm{H}), 4.77$ (d, 1 $\mathrm{H}, J 1.4 \mathrm{~Hz}), 4.39(\mathrm{dd}, 1 \mathrm{H}, J 3.3, J 9.9 \mathrm{~Hz})$, 4.35-4.33 (m, $2 \mathrm{H}), 4.24-4.22$ (m, $1 \mathrm{H}), 4.22-$ $4.20(\mathrm{~m}, 1 \mathrm{H}), 3.96-3.94(\mathrm{~m}, 1 \mathrm{H}), 3.89-3.74$ $(\mathrm{m}, 2 \mathrm{H}), 3.53-3.51(\mathrm{~m}, 1 \mathrm{H}), 1.91(\mathrm{~s}, 3 \mathrm{H}$, $\left.\mathrm{CH}_{3} \mathrm{CO}\right), 1.87$ (s, $\left.3 \mathrm{H}, \mathrm{CH}_{3} \mathrm{CO}\right), 1.82(\mathrm{~s}, 3 \mathrm{H}$, $\left.\mathrm{CH}_{3} \mathrm{CO}\right), 1.72-1.64(\mathrm{~m}, 2 \mathrm{H}), 1.43-1.24(\mathrm{~m}$, $16 \mathrm{H}), 1.03(\mathrm{~d}, 3 \mathrm{H}, J 6.1 \mathrm{~Hz}), 0.93-0.86(\mathrm{~m}$, $6 \mathrm{H}) ;{ }^{13} \mathrm{C} \mathrm{NMR}\left(\mathrm{CDCl}_{3}, 100 \mathrm{MHz}\right): \delta 14.1$, $17.1,17.3,17.6,17.7,20.5,20.5,20.7,22.7$, $26.1,26.2,29.3,29.4,29.5,31.8,67.0,67.2$, $67.5,67.6,68.2,68.5,69.5,70.5,71.1,71.7$, $71.8,71.9,72.0,73.1,74.6,77.0,77.7,98.9$, $99.1,99.3,100.8,165.3,165.4,165.5,165.7$, 165.9, 166.5, 169.2, 169.2, 170.0. Anal. Calcd for $\mathrm{C}_{80} \mathrm{H}_{88} \mathrm{O}_{26}: \mathrm{C}, 65.56 ; \mathrm{H}, 6.05$. Found: $\mathrm{C}$, 65.79; H, 6.04. 
Methyl $\alpha$-L-rhamnopyranosyl- $(1 \rightarrow 3)-\alpha-\mathrm{L}-$ rhamnopyranosyl- $(1 \rightarrow 2)-\alpha-\mathrm{L}$ - rhamnopyrano syl-(1 $\rightarrow 2)-\alpha$-L-rhamnopyranoside (22). - Tetrasaccharide 20 (680 $\mathrm{mg}, 0.5 \mathrm{mmol})$ was dissolved in a satd methanolic ammonia $(5 \mathrm{~mL})$. After $48 \mathrm{~h}$ at $\mathrm{rt}$, the reaction mixture was concentrated, and the residue was purified by chromatography on Sephadex LH-20 $(\mathrm{MeOH})$ to afford 22 as a foamy solid (248 $\mathrm{mg}, 80.5 \%)$ : $[\alpha]_{\mathrm{D}}+46^{\circ} \quad\left(c \quad 1.3, \mathrm{CH}_{3} \mathrm{OH}\right) .{ }^{1} \mathrm{H} \quad \mathrm{NMR}$ $\left(\mathrm{CD}_{3} \mathrm{OD}\right): \delta 5.08(\mathrm{~d}, 1 \mathrm{H}, J 1.3 \mathrm{~Hz}$, ), $5.05(\mathrm{~d}$, $1 \mathrm{H}, J 1.4 \mathrm{~Hz}), 4.94(\mathrm{~d}, 1 \mathrm{H}, J 1.3 \mathrm{~Hz}), 4.67(\mathrm{~d}$, $1 \mathrm{H}, J 1.3 \mathrm{~Hz}), 4.06(\mathrm{dd}, 1 \mathrm{H}, J 1.3, J 3.2 \mathrm{~Hz})$, $4.03\left(\mathrm{dd}, 1 \mathrm{H}, J 1.4, J_{23} 3.1 \mathrm{~Hz}\right), 3.98(\mathrm{dd}, 1$ $\mathrm{H}, J 1.5, J 3.2 \mathrm{~Hz}), 3.79-3.30(\mathrm{~m}, 16 \mathrm{H})$, $1.29-1.23(\mathrm{~m}, 12 \mathrm{H}) ;{ }^{13} \mathrm{C}$ NMR $\left(\mathrm{CD}_{3} \mathrm{OD}, 100\right.$ $\mathrm{MHz}): \delta 99.7,100.9,101.9,102.1,78.3,77.8$, 77.5, 53.4, 16.3, 16.3, 16.2, 16.0. MS $(\mathrm{m} / \mathrm{z})$ Calcd for $\mathrm{C}_{25} \mathrm{H}_{44} \mathrm{O}_{17}: 616$ [M]. Found: 639 $[\mathrm{M}+\mathrm{Na}]$.

Octyl $\alpha$ - L-rhamnopyranosyl- $(1 \rightarrow 3)-\alpha-\mathrm{L}-$ rhamnopyranosyl- $(1 \rightarrow 2)$ - $\alpha$ - L - rhamnopyrano -

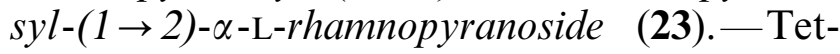
rasaccharide 21 (732 $\mathrm{mg}, 0.5 \mathrm{mmol}$ ) was dissolved in a satd methanolic ammonia $(5 \mathrm{~mL})$. After $48 \mathrm{~h}$ at $\mathrm{rt}$, the reaction mixture was concentrated, and the residue was purified by chromatography on Sephadex LH-20 $(\mathrm{MeOH})$ to afford 23 as a foamy solid (284 mg, 79.6\%): $[\alpha]_{\mathrm{D}}+36^{\circ} \quad(c \quad 1.3, \mathrm{MeOH}) ;{ }^{1} \mathrm{H} \quad \mathrm{NMR}$ $\left(\mathrm{CD}_{3} \mathrm{OD}\right): \delta 5.09(\mathrm{~d}, 1 \mathrm{H}, J 1.3 \mathrm{~Hz}), 5.05(\mathrm{~d}, 1$ $\mathrm{H}, J 1.3 \mathrm{~Hz}), 4.92(\mathrm{~d}, 1 \mathrm{H}, J 1.6 \mathrm{~Hz}), 4.77(\mathrm{~d}$, $1 \mathrm{H}, J 1.5 \mathrm{~Hz}$ ), 4.06 (dd, $1 \mathrm{H}, J 1.3, J 3.3 \mathrm{~Hz}$,), $4.02(\mathrm{dd}, 1 \mathrm{H}, J 1.5, J 3.3 \mathrm{~Hz}), 3.98(\mathrm{dd}, 1 \mathrm{H}$, $J 1.6, J 3.3 \mathrm{~Hz}), 3.82-3.30(\mathrm{~m}, 15 \mathrm{H}), 1.62-$ $1.53(\mathrm{~m}, 2 \mathrm{H}), 1.31-0.86(\mathrm{~m}, 25 \mathrm{H}) ;{ }^{13} \mathrm{C} \mathrm{NMR}$ $\left(\mathrm{CD}_{3} \mathrm{OD}, 100 \mathrm{MHz}\right): \delta$ 98.4, 100.9, 101.9, $102.1,78.5,77.8,77.5,31.2,28.7,28.6,28.5$, $25.5,21.9,16.3,16.2,16.1,16.0,12.6$. MS $(\mathrm{m} / \mathrm{z})$ Calcd for $\mathrm{C}_{32} \mathrm{H}_{58} \mathrm{O}_{17}: 714.5$ [M]. Found: $737.5[\mathrm{M}+\mathrm{Na}]$.

\section{Acknowledgements}

This work was supported by The Chinese Academy of Sciences (Projects KJ952J 510 and KIP-RCEES9904) and by The National Natural Science Foundation of China (Projects 29802009, 39970864, and 30070815).

\section{References}

1. Ansaruzzaman, M.; Albert, M. J.; Holme, T.; Jansson, P. E.; Rahman, M. M.; Widmalm, G. Eur. J. Biochem. 1996, 237, 786-791.

2. Knirel, Y. A.; Zdorovenko, G. M.; Shashkov, A. S.; Yakovleva, L. M.; Gubanova, N. Y.; Grozdyak, R. I. Bioorg. Khim. 1988, 14, 172-179.

3. (a) Wang, W.; Kong, F. J. Org. Chem. 1998, 63, 57445755 ;

(b) Zhu, Y.; Kong, F. Synlett 2000, 663-667.

4. Du, Y.; Kong, F. J. Carbohydr. Chem. 1999, 18, 655-664.

5. Schmidt, R. R.; Kinzy, W. Adv. Carbohydr. Chem. Biochem. 1994, 50, 21-125.

6. Zhu, Y.; Kong, F. Synlett 2000, 1783-1788.

7. (a) Auzanneau, F.-I.; Forooghian, F.; Pinto, B. M. Carbohydr. Res. 1996, 291, 21-30;

(b) Wang, W.; Kong, F. Carbohydr. Res. 1999, 315, 128135. 